ISSN 1855-3966 (printed edn.), ISSN 1855-3974 (electronic edn.)

ARS MATHEMATICA CONTEMPORANEA 16 (2019) 81-95

https://doi.org/10.26493/1855-3974.1333.68f

(Also available at http://amc-journal.eu)

\title{
Pentavalent symmetric graphs of order four times an odd square-free integer*
}

\author{
Bo Ling ${ }^{\dagger}$ \\ School of Mathematics and Computer Sciences, Yunnan Minzu University, \\ Kunming, Yunnan 650504, P. R. China \\ Ben Gong Lou \\ School of Mathematics and Statistics, Yunnan University, \\ Kunming, Yunnan 650031, P. R. China \\ Ci Xuan $\mathrm{Wu}$ \\ School of Statistics and Mathematics, Yunnan University of Finance and Economics, \\ Kunming, Yunnan 650221, P. R. China
}

Received 24 February 2017, accepted 6 June 2018, published online 18 September 2018

\begin{abstract}
A graph is said to be symmetric if its automorphism group is transitive on its arcs. Guo et al. in 2011 and Pan et al. in 2013 determined all pentavalent symmetric graphs of order $4 p q$. In this paper, we shall generalize this result by determining all connected pentavalent symmetric graphs of order four times an odd square-free integer. It is shown in this paper that, for each such graph $\Gamma$, either the full automorphism group Aut $\Gamma$ is isomorphic to $\operatorname{PSL}(2, p), \operatorname{PGL}(2, p), \operatorname{PSL}(2, p) \times \mathbb{Z}_{2}$ or $\operatorname{PGL}(2, p) \times \mathbb{Z}_{2}$, or $\Gamma$ is isomorphic to one of 9 graphs.
\end{abstract}

Keywords: Arc-transitive graph, normal quotient, automorphism group.

Math. Subj. Class.: 05C25, 05E18, 20B25

\footnotetext{
* The authors are very grateful to the referee for the constructive comments and suggestions. The first author was supported by the National Natural Science Foundation of China (11701503, 11861076, 11761079), Yunnan Applied Basic Research Projects (2018FB003) and the Scientific Research Foundation Project of Yunnan Education Department (2017ZZX086). The second author was supported by the National Natural Science Foundation of China (11861076, 11231008, 11461004, 11301468).

†Corresponding author.

E-mail addresses: bolinggxu@163.com (Bo Ling), bengong188@163.com (Ben Gong Lou), wucixuan@gmail.com (Ci Xuan Wu)
} 


\section{Introduction}

All graphs in this paper are assumed to be finite, simple and undirected. Let $\Gamma$ be a graph and denote $V \Gamma$ and $A \Gamma$ the vertex set and arc set of $\Gamma$, respectively. Let $G$ be a subgroup of the full automorphism group Aut $\Gamma$ of $\Gamma$. Then $\Gamma$ is called $G$-vertex-transitive and $G$ arc-transitive if $G$ is transitive on $V \Gamma$ and $A \Gamma$, respectively. An arc-transitive graph is also called a symmetric graph. It is well known that $\Gamma$ is $G$-arc-transitive if and only if $G$ is transitive on $V \Gamma$ and the stabilizer $G_{\alpha}:=\left\{g \in G \mid \alpha^{g}=\alpha\right\}$ is transitive on the neighbor set $\Gamma(\alpha)$ of the vertex $\alpha$ of $\Gamma$.

The cubic and tetravalent graphs have been studied extensively in the literature. In recent years, attention has moved on to pentavalent symmetric graphs and a series of results have been obtained. For example, all the possibilities of vertex stabilizers of pentavalent symmetric graphs are determined in [7, 20]. Also, for distinct primes $p, q$ and $r$, the classifications of pentavalent symmetric graphs of order $2 p q$ and $2 p q r$ are presented in [9, 19], respectively. A classification of 1-regular pentavalent graph (that is, the full automorphism group acts regularly on its arc set) of square-free order is presented in [13]. Recently, pentavalent symmetric graphs of square-free order have been completely classified in [11]. Furthermore, some classifications of pentavalent symmetric graphs of cube-free order also have been obtained in recent years. For example, the classifications of pentavalent symmetric graphs of order $12 p, 4 p q$ and $2 p^{2}$ are presented in [8, 16, 5]. More recently, symmetric graphs of any prime valency which admit a soluble arc-transitive group have been classified in [14]. The main purpose of this paper is to extend the results in [8, 16] to four times an odd square-free integer case.

The main result of this paper is the following theorem.

Theorem 1.1. Let $n$ be an odd square-free integer and let $\Gamma$ be a pentavalent symmetric graph of order $4 n$. If $n$ has at least three prime factors, then one of the following statements holds.

(1) Aut $\Gamma \cong \operatorname{PSL}(2, p), \operatorname{PGL}(2, p), \operatorname{PSL}(2, p) \times \mathbb{Z}_{2}$ or $\operatorname{PGL}(2, p) \times \mathbb{Z}_{2}$, where $p \geq 29$ is a prime. Furthermore, the stabilizer $(\text { Aut } \Gamma)_{\alpha}$ and the prime $p$ appear in Table 5 or Table 6.

(2) The triple $(\Gamma, n$, Aut $\Gamma$ ) lies in the following Table 1.

Remark 1.2 (Remarks on Theorem 1.1).

(a) The graphs in Table 1 are introduced in Example 3.2.

(b) The graphs $\mathcal{C}_{5852}$ and $\mathcal{C}_{780}^{3}$ in Table 1, and the graphs in part (1) with automorphism group $\operatorname{PSL}(2, p) \times \mathbb{Z}_{2}$ or $\operatorname{PGL}(2, p) \times \mathbb{Z}_{2}$ can also be constructed from the bipartite double cover (the definition of bipartite double cover see Section 3) of a pentavalent symmetric graph of square-free order (see [11, Example 4.3 and Example 4.5] and [19, Example 3.9 and Example 3.11] for details on these graphs).

\section{Preliminaries}

We now give some necessary preliminary results. The first one is a property of the Fitting subgroup, see [18, p. 30, Corollary].

Lemma 2.1. Let $F$ be the Fitting subgroup of a group $G$. If $G$ is soluble, then $F \neq 1$ and the centralizer $\mathbf{C}_{G}(F) \leq F$. 
Table 1: Nine 'sporadic' pentavalent symmetric graphs of order four times an odd squarefree integer.

\begin{tabular}{ccccccc} 
Row & $\Gamma$ & $n$ & Aut $\Gamma$ & $(\text { Aut } \Gamma)_{\alpha}$ & Transitivity & Bipartite? \\
\hline \hline 1 & $\mathcal{C}_{17556}^{1}$ & $3 \cdot 7 \cdot 11 \cdot 19$ & $\mathrm{~J}_{1}$ & $\mathrm{D}_{10}$ & 1-transitive & No \\
2 & $\mathcal{C}_{17556}^{2}$ & $3 \cdot 7 \cdot 11 \cdot 19$ & $\mathrm{~J}_{1}$ & $\mathrm{D}_{10}$ & 1-transitive & No \\
3 & $\mathcal{C}_{17556}^{3}$ & $3 \cdot 7 \cdot 11 \cdot 19$ & $\mathrm{~J}_{1}$ & $\mathrm{D}_{10}$ & 1-transitive & No \\
4 & $\mathcal{C}_{17556}^{4}$ & $3 \cdot 7 \cdot 11 \cdot 19$ & $\mathrm{~J}_{1}$ & $\mathrm{D}_{10}$ & 1-transitive & No \\
5 & $\mathcal{C}_{17556}^{5}$ & $3 \cdot 7 \cdot 11 \cdot 19$ & $\mathrm{~J}_{1}$ & $\mathrm{D}_{10}$ & 1-transitive & No \\
6 & $\mathcal{C}_{5852}$ & $7 \cdot 11 \cdot 19$ & $\mathrm{~J}_{1} \times \mathbb{Z}_{2}$ & $\mathrm{~A}_{5}$ & 2-transitive & Yes \\
7 & $\mathcal{C}_{780}^{1}$ & $3 \cdot 5 \cdot 13$ & $\operatorname{PSL}(2,25) \times \mathbb{Z}_{2}$ & $\mathrm{~F}_{20}$ & 2-transitive & No \\
8 & $\mathcal{C}_{780}^{2}$ & $3 \cdot 5 \cdot 13$ & $\operatorname{PSL}(2,25) \times \mathbb{Z}_{2}$ & $\mathrm{~F}_{20}$ & 2-transitive & No \\
9 & $\mathcal{C}_{780}^{3}$ & $3 \cdot 5 \cdot 13$ & $\operatorname{PSL}(2,25) \times \mathbb{Z}_{2}$ & $\mathrm{~F}_{20}$ & 2-transitive & Yes
\end{tabular}

The maximal subgroups of PSL(2, $p)$ are known, see [4, Section 239].

Lemma 2.2. Let $T=\operatorname{PSL}(2, p)$, where $p \geq 5$ is a prime. Then a maximal subgroup of $T$ is isomorphic to one of the following groups:

(1) $\mathrm{D}_{p-1}$, where $p \neq 5,7,9,11$;

(2) $\mathrm{D}_{p+1}$, where $p \neq 7,9$;

(3) $\mathbb{Z}_{p}: \mathbb{Z}_{(p-1) / 2}$;

(4) $\mathrm{A}_{4}$, where $p=5$ or $p \equiv 3,13,27,37(\bmod 40)$;

(5) $\mathrm{S}_{4}$, where $p \equiv \pm 1(\bmod 8)$

(6) $\mathrm{A}_{5}$, where $p \equiv \pm 1(\bmod 5)$.

By [2, Theorem 2], we may easily derive the maximal subgroups of $\operatorname{PGL}(2, p)$.

Lemma 2.3. Let $T=\operatorname{PGL}(2, p)$ with $p \geq 5$ a prime. Then a maximal subgroup of $T$ is isomorphic to one of the following groups:

(1) $\mathbb{Z}_{p}: \mathbb{Z}_{p-1}$;

(2) $\mathrm{D}_{2(p+1)}$;

(3) $\mathrm{D}_{2(p-1)}$, where $p \geq 7$;

(4) $\mathrm{S}_{4}$, where $p \equiv \pm 3(\bmod 8)$;

(5) $\operatorname{PSL}(2, p)$.

From [6, pp. 134-136], we can obtain the following lemma by checking the orders of nonabelian simple groups.

Lemma 2.4. Let $n$ be an odd square-free integer such that $n$ has at least three prime factors. Let $T$ be a nonabelian simple group of order $2^{i} \cdot 3^{j} \cdot 5 \cdot n$, where $1 \leq i \leq 11$ and $0 \leq j \leq 2$. Let $p$ be the largest prime factor of $n$. Then $T$ is listed in Table 2. 
Table 2: Nonabelian simple groups of order $2^{i} \cdot 3^{j} \cdot 5 \cdot n$ with $1 \leq i \leq 11$ and $0 \leq j \leq 2$.

\begin{tabular}{lll}
$T$ & $|T|$ & $n$ \\
\hline \hline $\mathrm{M}_{22}$ & $2^{7} \cdot 3^{2} \cdot 5 \cdot 7 \cdot 11$ & $3 \cdot 7 \cdot 11$ \\
$\mathrm{M}_{23}$ & $2^{7} \cdot 3^{2} \cdot 5 \cdot 7 \cdot 11 \cdot 23$ & $7 \cdot 11 \cdot 23$ \\
$\mathrm{~J}_{1}$ & $2^{3} \cdot 3 \cdot 5 \cdot 7 \cdot 11 \cdot 19$ & $7 \cdot 11 \cdot 19$ \\
$\mathrm{~J}_{2}$ & $2^{7} \cdot 3^{3} \cdot 5^{2} \cdot 7$ & $3 \cdot 5 \cdot 7$ \\
$\mathrm{Sz}(32)$ & $2^{10} \cdot 5^{2} \cdot 31 \cdot 41$ & $5 \cdot 31 \cdot 41$ \\
$\operatorname{PSU}(3,4)$ & $2^{6} \cdot 3 \cdot 5^{2} \cdot 13$ & $3 \cdot 5 \cdot 13$ \\
$\operatorname{PSp}(4,4)$ & $2^{8} \cdot 3^{2} \cdot 5^{2} \cdot 17$ & $3 \cdot 5 \cdot 17$ \\
$\operatorname{PSL}(2,25)$ & $2^{3} \cdot 3 \cdot 5^{2} \cdot 13$ & $3 \cdot 5 \cdot 13$ \\
$\operatorname{PSL}\left(2,2^{8}\right)$ & $2^{8} \cdot 3 \cdot 5 \cdot 17 \cdot 257$ & $3 \cdot 17 \cdot 257$ \\
$\operatorname{PSL}(5,2)$ & $2^{10} \cdot 3^{2} \cdot 5 \cdot 7 \cdot 31$ & $3 \cdot 7 \cdot 31$ \\
$\operatorname{PSL}\left(2,2^{6}\right)$ & $2^{6} \cdot 3^{2} \cdot 5 \cdot 7 \cdot 13$ & $3 \cdot 7 \cdot 13$ \\
\hline $\mathrm{M}_{23}$ & $2^{7} \cdot 3^{2} \cdot 5 \cdot 7 \cdot 11 \cdot 23$ & $3 \cdot 7 \cdot 11 \cdot 23$ \\
$\mathrm{M}_{24}$ & $2^{10} \cdot 3^{3} \cdot 5 \cdot 7 \cdot 11 \cdot 23$ & $3 \cdot 7 \cdot 11 \cdot 23$ \\
$\mathrm{~J}_{1}$ & $2^{3} \cdot 3 \cdot 5 \cdot 7 \cdot 11 \cdot 19$ & $3 \cdot 7 \cdot 11 \cdot 19$ \\
\hline $\operatorname{PSL}(2, p)$ & $\frac{p(p+1)(p-1)}{2} \quad(p \geq 29)$ &
\end{tabular}

Proof. If $T$ is a sporadic simple group, by [6, p. 135-136], $T=\mathrm{M}_{22}, \mathrm{M}_{23}, \mathrm{M}_{24}, \mathrm{~J}_{1}$ or $\mathrm{J}_{2}$. If $T=\mathrm{A}_{n}$ is an alternating group, since $3^{4}$ does not divide $|T|$, we have $n \leq 8$, it then easily exclude that $T=\mathrm{A}_{5}, \mathrm{~A}_{6}, \mathrm{~A}_{7}$ or $\mathrm{A}_{8}$. Hence no $T$ exists for this case.

Suppose now $T=X(q)$ is a simple group of Lie type, where $X$ is one type of Lie groups, and $q=r^{d}$ is a prime power. If $r \geq 5$, as $|T|$ has at most three 3 -factors, two 5 -factors and one $p$-factor, it easily follows from [6, p. 135] that the only possibility is $T=\operatorname{PSL}(2, p)$ with $p \geq 29$ (note that $\operatorname{PSL}(2, p)$ with $5 \leq p \leq 23$ does not satisfy the condition of the lemma) or $\operatorname{PSL}(2,25)$, where $p$ is the largest prime factor of $n$. If $r \leq 3$, as $2^{12}$ and $3^{4}$ do not divide $|T|$, then we have $T=\operatorname{Sz}(32), \operatorname{PSU}(3,4), \operatorname{PSp}(4,4), \operatorname{PSL}\left(2,2^{6}\right)$, $\operatorname{PSL}\left(2,2^{8}\right)$ or $\operatorname{PSL}(5,2)$.

For a graph $\Gamma$ and a positive integer $s$, an $s$ - $\operatorname{arc}$ of $\Gamma$ is a sequence $\alpha_{0}, \alpha_{1}, \ldots, \alpha_{s}$ of vertices such that $\alpha_{i-1}, \alpha_{i}$ are adjacent for $1 \leq i \leq s$ and $\alpha_{i-1} \neq \alpha_{i+1}$ for $1 \leq i \leq s-1$. In particular, a 1 -arc is just an arc. Then $\Gamma$ is called $(G, s)$-arc-transitive with $G \leq$ Aut $\Gamma$ if $G$ is transitive on the set of $s$-arcs of $\Gamma$. A $(G, s)$-arc-transitive graph is called $(G, s)$ transitive if it is not $(G, s+1)$-arc-transitive. In particular, a graph $\Gamma$ is simply called $s$-transitive if it is (Aut $\Gamma, s$ )-transitive.

Let $\mathrm{F}_{20}$ denote the Frobenius group of order 20. The following lemma determines the stabilizers of pentavalent symmetric graphs, refer to $[7,20]$.

Lemma 2.5. Let $\Gamma$ be a pentavalent $(G, s)$-transitive graph, where $G \leq$ Aut $\Gamma$ and $s \geq 1$. Let $\alpha \in V \Gamma$. Then one of the following holds.

(a) If $G_{\alpha}$ is soluble, then $s \leq 3$ and $\left|G_{\alpha}\right| \mid 80$. Further, the pair $\left(s, G_{\alpha}\right)$ lies in the 
following table.

\begin{tabular}{c|c}
$s$ & $G_{\alpha}$ \\
\hline \hline 1 & $\mathbb{Z}_{5}, \mathrm{D}_{10}, \mathrm{D}_{20}$ \\
2 & $\mathrm{~F}_{20}, \mathrm{~F}_{20} \times \mathbb{Z}_{2}$ \\
3 & $\mathrm{~F}_{20} \times \mathbb{Z}_{4}$
\end{tabular}

(b) If $G_{\alpha}$ is insoluble, then $2 \leq s \leq 5$, and $\left|G_{\alpha}\right| \mid 2^{9} \cdot 3^{2} \cdot 5$. Further, the pair $\left(s, G_{\alpha}\right)$ lies in the following table.

\begin{tabular}{c|c|c}
$s$ & $G_{\alpha}$ & $\left|G_{\alpha}\right|$ \\
\hline \hline 2 & $\mathrm{~A}_{5}, \mathrm{~S}_{5}$ & 60,120 \\
3 & $\mathrm{~A}_{4} \times \mathrm{A}_{5},\left(\mathrm{~A}_{4} \times \mathrm{A}_{5}\right): \mathbb{Z}_{2}, \mathrm{~S}_{4} \times \mathrm{S}_{5}$ & $720,1440,2880$ \\
4 & $\mathrm{ASL}(2,4), \mathrm{AGL}(2,4), \mathrm{A} \Sigma \mathrm{L}(2,4), \mathrm{A} \Gamma \mathrm{L}(2,4)$ & $960,1920,2880,5760$ \\
5 & $\mathbb{Z}_{2}^{6}: \Gamma \mathrm{TL}(2,4)$ & 23040
\end{tabular}

A typical method for studying vertex-transitive graphs is taking normal quotients. Let $\Gamma$ be a $G$-vertex-transitive graph, where $G \leq$ Aut $\Gamma$. Suppose that $G$ has a normal subgroup $N$ which is intransitive on $V \Gamma$. Let $V \Gamma_{N}$ be the set of $N$-orbits on $V \Gamma$. The normal quotient graph $\Gamma_{N}$ of $\Gamma$ induced by $N$ is defined as the graph with vertex set $V \Gamma_{N}$, and $B$ is adjacent to $C$ in $\Gamma_{N}$ if and only if there exist vertices $\beta \in B$ and $\gamma \in C$ such that $\beta$ is adjacent to $\gamma$ in $\Gamma$. In particular, if $\operatorname{val}(\Gamma)=\operatorname{val}\left(\Gamma_{N}\right)$, then $\Gamma$ is called a normal cover of $\Gamma_{N}$.

A graph $\Gamma$ is called $G$-locally primitive if, for each $\alpha \in V \Gamma$, the stabilizer $G_{\alpha}$ acts primitively on $\Gamma(\alpha)$. Obviously, a pentavalent symmetric graph is locally primitive. The following theorem gives a basic method for studying vertex-transitive locally primitive graphs, see [17, Theorem 4.1] and [12, Lemma 2.5].

Theorem 2.6. Let $\Gamma$ be a $G$-vertex-transitive locally primitive graph, where $G \leq$ Aut $\Gamma$, and let $N \triangleleft G$ have at least three orbits on $V \Gamma$. Then the following statements hold.

(i) $N$ is semi-regular on $V \Gamma, G / N \leq$ Aut $\Gamma_{N}$, and $\Gamma$ is a normal cover of $\Gamma_{N}$;

(ii) $G_{\alpha} \cong(G / N)_{\gamma}$, where $\alpha \in V \Gamma$ and $\gamma \in V \Gamma_{N}$;

(iii) $\Gamma$ is $(G, s)$-transitive if and only if $\Gamma_{N}$ is $(G / N, s)$-transitive, where $1 \leq s \leq 5$ or $s=7$.

For reduction, we need some information of pentavalent symmetric graphs of order $4 p q$, stated in the following lemma, see [8, Theorem 4.1] and [16, Theorem 3.1].

Lemma 2.7. Let $\Gamma$ be a pentavalent symmetric graph of order $4 p q$, where $q>p \geq 3$ are primes. Then the pair (Aut $\left.\Gamma,(\text { Aut } \Gamma)_{\alpha}\right)$ lies in the following Table 3, where $\alpha \in V \Gamma$.

Remark 2.8 (Remarks on Lemma 2.7).

(a) Suppose that $\Gamma$ is one of the graphs in Lemma 2.7 and $M$ is an arc-transitive subgroup of Aut $\Gamma$. Then $M$ is insoluble (for convenience, we prove this conclusion in Lemma 4.4 and we remark that Lemma 4.4 is independent where it is used).

(b) By MAGMA [1], the graphs $\mathcal{C}_{66}^{(2)}$ and $\mathcal{C}_{132}^{5}$ in [8, Theorem 4.1] are isomorphic, $\operatorname{Aut}\left(\mathcal{C}_{132}^{5}\right) \cong \operatorname{PGL}(2,11) \times \mathbb{Z}_{2}$. 
Table 3: Pentavalent symmetric graphs of order $4 p q$.

\begin{tabular}{llll}
$\Gamma$ & $(p, q)$ & $\operatorname{Aut} \Gamma$ & $(\text { Aut } \Gamma)_{\alpha}$ \\
\hline \hline $\mathcal{C}_{60}$ & $(3,5)$ & $\mathrm{A}_{5} \times \mathrm{D}_{10}$ & $\mathrm{D}_{10}$ \\
$\mathcal{C}_{132}^{1}$ & $(3,11)$ & $\operatorname{PSL}(2,11) \times \mathbb{Z}_{2}$ & $\mathrm{D}_{10}$ \\
$\mathcal{C}_{132}^{i}, 2 \leq i \leq 4$ & $(3,11)$ & $\operatorname{PGL}(2,11)$ & $\mathrm{D}_{10}$ \\
$\mathcal{C}_{132}^{5}$ & $(3,11)$ & $\operatorname{PGL}(2,11) \times \mathbb{Z}_{2}$ & $\mathrm{D}_{20}$ \\
$\mathcal{C}_{574}^{(2)}$ & $(7,41)$ & $\operatorname{PSL}(2,41) \times \mathbb{Z}_{2}$ & $\mathrm{~A}_{5}$ \\
$\mathcal{C}_{4108}$ & $(13,79)$ & $\operatorname{PSL}(2,79)$ & $\mathrm{A}_{5}$
\end{tabular}

The final lemma of this section gives some information about the pentavalent symmetric graphs of square-free order, refer to [19, Theorem 1.1] and [11, Theorem 1.1].

Lemma 2.9. Let $\Gamma$ be a pentavalent symmetric graph of order $2 n$, where $n$ is an odd square-free integer and has at least three prime factors. Then one of the following statements holds.

(1) Aut $\Gamma$ is soluble and Aut $\Gamma \cong \mathrm{D}_{2 n}: \mathbb{Z}_{5}$.

(2) Aut $\Gamma=\operatorname{PSL}(2, p)$ or $\operatorname{PGL}(2, p)$, where $p \geq 5$ is a prime.

(3) The triple $(\Gamma, 2 n$, Aut $\Gamma)$ lies in the following Table 4.

Table 4: Two 'sporadic' pentavalent symmetric graphs.

\begin{tabular}{llll}
$\Gamma$ & $2 n$ & Aut $\Gamma$ & $(\text { Aut } \Gamma)_{\alpha}$ \\
\hline \hline $\mathcal{C}_{390}$ & 390 & $\operatorname{PSL}(2,25)$ & $\mathrm{F}_{20}$ \\
$\mathcal{C}_{2926}$ & 2926 & $\mathrm{~J}_{1}$ & $\mathrm{~A}_{5}$
\end{tabular}

\section{Some examples}

In this section, we give some examples of pentavalent symmetric graphs of order $4 n$ with $n$ an odd square-free integer.

In order to construct our graphs we first introduce the definition of a coset graph. Let $G$ be a finite group and let $H$ be a core-free subgroup of $G$. Let $\tau \in G$ and $\tau^{2} \in H$. Define the coset graph $\operatorname{Cos}(G, H, \tau)$ of $G$ with respect to $H$ as the graph with vertex set $[G: H]$ such that $H x, H y$ are adjacent if and only if $y x^{-1} \in H \tau H$. The following lemma about coset graphs is well known and the proof of the lemma follows from the definition of coset graphs.

Lemma 3.1. Using the notation as above, the coset graph $\Gamma=\operatorname{Cos}(G, H, \tau)$ is $G$-arctransitive graph and

(1) $\operatorname{val} \Gamma=\left|H: H \cap H^{\tau}\right|$;

(2) $\Gamma$ is connected if and only if $\langle H, \tau\rangle=G$. 
Conversely, each $G$-arc-transitive graph $\Sigma$ is isomorphic to the coset graph $\operatorname{Cos}\left(G, G_{v}, \tau\right)$, where $\tau \in \mathbf{N}_{G}\left(G_{v w}\right)$ is a 2-element such that $\tau^{2} \in G_{v}$, and $v \in V \Sigma, w \in \Sigma(v)$.

We next introduce the definition of the bipartite double cover of a graph. Let $\Gamma$ be a graph with vertex set $V \Gamma$. The standard double cover of $\Gamma$ is defined as the undirected bipartite graph $\widetilde{\Gamma}$ with biparts $V_{0}$ and $V_{1}$, where $V_{i}=\{(v, i) \mid v \in V \Gamma\}$, such that two vertices $(x, 0)$ and $(y, 1)$ are adjacent if and only if $x, y$ are adjacent in $\Gamma$. It is easily shown that the standard double cover can be represented as a direct product: $\widetilde{\Gamma}=\Gamma \times \mathrm{K}_{2}$. Furthermore, $\widetilde{\Gamma}$ is connected if and only if $\Gamma$ is connected and non-bipartite.

For a given small permutation group $X$, we may determine all graphs which admit $X$ as an arc-transitive automorphism group by using MAGMA [1]. It is then easy to have the following result.

\section{Example 3.2.}

(1) There is a unique pentavalent symmetric graph of order 5852 which admits $J_{1} \times \mathbb{Z}_{2}$ as an arc-transitive automorphism group; and its full automorphism group is $J_{1} \times \mathbb{Z}_{2}$. This graph is denoted by $\mathcal{C}_{5832}$ which satisfies the conditions in Row 6 of Table 1.

(2) There are five pentavalent symmetric graphs of order 17556 admitting $\mathrm{J}_{1}$ as an arctransitive automorphism group; and their full automorphism group are all isomorphic to $\mathrm{J}_{1}$. These five graphs are denoted by $\mathcal{C}_{17556}^{i}$ which satisfy the conditions in Row 1 to Row 5 of Table 1 , where $1 \leq i \leq 5$.

(3) There are three pentavalent symmetric graphs of order 780 which admit $\operatorname{PSL}(2,25) \times$ $\mathbb{Z}_{2}$ as an arc-transitive automorphism group; and their full automorphism group are all isomorphic to $\operatorname{PSL}(2,25) \times \mathbb{Z}_{2}$. These three graphs are denoted by $\mathcal{C}_{780}^{j}$ which satisfy the conditions in Row 7 to Row 9 of Table 1 , where $1 \leq j \leq 3$.

Remark 3.3 (Remarks on Example 3.2).

(a) Let $\Gamma$ be a pentavalent symmetric graph of order $4 n$ with $n$ an odd square-free integer and having at least three prime factors. Then the graphs appearing in Example 3.2 are the only sporadic graphs of such $\Gamma$. In fact, let $\mathrm{A}=$ Aut $\Gamma$. If $\mathrm{A}$ is insoluble and has no nontrivial soluble normal subgroup, then Lemma 4.2 shows that $\mathcal{C}_{17556}^{i}$ with $1 \leq i \leq 5$ are the only sporadic graphs. If $\mathrm{A}$ is insoluble and has a soluble minimal normal subgroup $N=\mathbb{Z}_{2}$, then Lemma 4.3 shows that $\mathcal{C}_{5832}$ and $\mathcal{C}_{780}^{j}$ with $1 \leq j \leq 3$ are the only sporadic graphs. If $\mathrm{A}$ is soluble or has a soluble minimal normal subgroup $N=\mathbb{Z}_{r}$ with $r>2$, then Lemma 4.1 and Lemma 4.6 show that no such $\Gamma$ exists.

(b) Since both $\mathcal{C}_{2926}$ and $\mathcal{C}_{390}$ are non-bipartite, the bipartite double cover of both $\mathcal{C}_{2926}$ and $\mathcal{C}_{390}$ is connected pentavalent symmetric graph of order $4 n$. In fact, the graph $\mathcal{C}_{5832}$ is isomorphic to the bipartite double cover of $\mathcal{C}_{2926}$ and the graph $\mathcal{C}_{780}^{3}$ is isomorphic to the bipartite double cover of $\mathcal{C}_{390}$.

Example 3.4. Let $p$ be a prime such that

$$
p \equiv 49,79,81,111 \quad(\bmod 160)
$$

and let $\mathrm{A}=\operatorname{PSL}(2, p)$. Then by Lemma 2.2, $\mathrm{A}$ has a subgroup $H \cong \mathrm{A}_{5}$. Let $K<H$ with $K \cong \mathrm{A}_{4}$. Then $\mathbf{N}_{\mathrm{A}}(K)=K:\langle\tau\rangle \cong \mathrm{S}_{4}$, where $\tau \in \mathrm{A}-H$ is an involution. Let $\Gamma=\operatorname{Cos}(\mathrm{A}, H, H \tau H)$. Then $\Gamma$ is a connected pentavalent symmetric graph. 
Example 3.5. Let $p$ be a prime such that

$$
p \equiv 9,39,41,71 \quad(\bmod 80)
$$

and let $\mathrm{A}=\operatorname{PGL}(2, p)$. Then by Lemma 2.2 and Lemma 2.3 , A has a subgroup $H \cong \mathrm{A}_{5}$. Let $K<H$ with $K \cong \mathrm{A}_{4}$. Then $\mathrm{N}_{\mathrm{A}}(K)=K:\langle\tau\rangle \cong \mathrm{S}_{4}$ is a maximal subgroup of A, where $\tau \in \mathrm{A}-H$ is an involution, and so $\langle H, \tau\rangle=\mathrm{A}$. Let $\Gamma=\operatorname{Cos}(\mathrm{A}, H, H \tau H)$. Then $\Gamma$ is a connected pentavalent symmetric graph.

Example 3.6. Let $p$ be a prime such that

$$
p \equiv 9,39,41,71 \quad(\bmod 80)
$$

and let $\mathrm{A}=\operatorname{PSL}(2, p) \times \mathbb{Z}_{2}=T \times\langle z\rangle$, where $T=\operatorname{PSL}(2, p)$ and $\langle z\rangle=\mathbb{Z}_{2}$. Then $T$ has a subgroup $H \cong \mathrm{A}_{5}$. Let $K<H$ with $K \cong \mathrm{A}_{4}$. Then $\mathbf{N}_{\mathrm{A}}(K)=K:\langle\tau\rangle \times\langle z\rangle \cong \mathrm{S}_{4} \times \mathbb{Z}_{2}$, where $\tau \in T-H$ is an involution. Let $\Gamma=\operatorname{Cos}(\mathrm{A}, H, H \tau z H)$. Then $\Gamma$ is a connected pentavalent symmetric graph.

Example 3.7. Let $p$ be a prime such that

$$
p \equiv 11,19,21,29 \quad(\bmod 40)
$$

and let $\mathrm{A}=\operatorname{PGL}(2, p) \times \mathbb{Z}_{2}=T \times\langle z\rangle$, where $T=\operatorname{PGL}(2, p)$ and $\langle z\rangle=\mathbb{Z}_{2}$. Then $T$ has a subgroup $H \cong \mathrm{A}_{5}$. Let $K<H$ with $K \cong \mathrm{A}_{4}$. Then $\mathbf{N}_{\mathrm{A}}(K)=K:\langle\tau\rangle \times\langle z\rangle \cong \mathrm{S}_{4} \times \mathbb{Z}_{2}$, where $\tau \in T-H$ is an involution. Let $\Gamma=\operatorname{Cos}(\mathrm{A}, H, H \tau z H)$. Then $\Gamma$ is a connected pentavalent symmetric graph.

\section{Proof of Theorem 1.1}

Let $n$ be an odd square-free integer and $n$ has at least three prime factors. Let $\Gamma$ be a pentavalent symmetric graph of order $4 n$. Set $\mathrm{A}=$ Aut $\Gamma$. By Lemma $2.5,\left|\mathrm{~A}_{\alpha}\right| \mid 2^{9} \cdot 3^{2} \cdot 5$, and hence $|\mathrm{A}| \mid 2^{11} \cdot 3^{2} \cdot 5 \cdot n$. Assume that $n=p_{1} p_{2} \cdots p_{s}$, where $s \geq 3$ and $p_{i}$ 's are distinct primes.

Lemma 4.1. The group A is insoluble.

Proof. Suppose to the contrary that A is soluble. Let $F$ be the Fitting subgroup of A. By Lemma $2.1, F \neq 1$ and $\mathbf{C}_{\mathrm{A}}(F) \leq F$. Further, $F=\mathbf{O}_{2}(\mathrm{~A}) \times \mathbf{O}_{p_{1}}(\mathrm{~A}) \times \mathbf{O}_{p_{2}}(\mathrm{~A}) \times \cdots \times$ $\mathbf{O}_{p_{s}}(\mathrm{~A})$, where $\mathbf{O}_{2}(\mathrm{~A}), \mathbf{O}_{p_{1}}(\mathrm{~A}), \mathbf{O}_{p_{2}}(\mathrm{~A}), \ldots, \mathbf{O}_{p_{s}}(\mathrm{~A})$ denote the largest normal 2-, $p_{1^{-}}$, $p_{2^{-}}, \ldots, p_{s}$-subgroups of $\mathrm{A}$, respectively.

For each $p_{i} \in\left\{p_{1}, p_{2}, \ldots, p_{s}\right\}, \mathbf{O}_{p_{i}}(\mathrm{~A})$ has at least three orbits on $V \Gamma$, by Theorem 2.6, $\mathbf{O}_{p_{i}}(\mathrm{~A})$ is semi-regular on $V \Gamma$. Therefore, $F$ is semi-regular on $V \Gamma$ and so $|F|$ divides $|V \Gamma|=4 n$. Since $n=p_{1} p_{2} \cdots p_{s}$, we have $\mathbf{O}_{p_{i}}(\mathrm{~A}) \leq \mathbb{Z}_{p_{i}}$. This argument also proves $\mathbf{O}_{2}(\mathrm{~A}) \leq \mathbb{Z}_{4}$ or $\mathbb{Z}_{2}^{2}$. If $\mathbf{O}_{2}(\mathrm{~A})=\mathbb{Z}_{4}$ or $\mathbb{Z}_{2}^{2}$, then by Theorem 2.6 , the normal quotient graph $\Gamma_{\mathbf{O}_{2}}$ (A) is a pentavalent symmetric graph of odd order, which is a contradiction. Thus, $\mathbf{O}_{2}(\mathrm{~A}) \leq \mathbb{Z}_{2}, F \cong \mathbb{Z}_{m}$, where $m \mid 2 n$. It implies that $\mathbf{C}_{\mathrm{A}}(F) \geq F$, and so $\mathbf{C}_{\mathrm{A}}(F)=F$.

If $F$ has at least three orbits on $V \Gamma$, then, by Theorem 2.6, $\Gamma_{F}$ is $\mathrm{A} / F$-arc-transitive. Since $\mathrm{A} / F=\mathrm{A} / \mathbf{C}_{\mathrm{A}}(F) \leq \operatorname{Aut}(F)$ is abelian, we have $(\mathrm{A} / F)_{\delta}=1$, where $\delta \in V \Gamma_{F}$, which is a contradiction. 
Thus, $F$ has at most two orbits on $V \Gamma$. If $F$ is transitive on $V \Gamma$, then $F$ is regular on $V \Gamma$, a contradiction with $F \cong \mathbb{Z}_{m}$, where $m \mid 2 n$. Hence $F$ has two orbits on $V \Gamma$ and $F \cong \mathbb{Z}_{2 n}$. Let $K=\mathbf{O}_{p_{3}}(\mathrm{~A}) \times \mathbf{O}_{p_{4}}(\mathrm{~A}) \times \cdots \times \mathbf{O}_{p_{s}}(\mathrm{~A})$. Then $K \cong \mathbb{Z}_{p_{3} p_{4} \cdots p_{s}}$. Since $K \unlhd \mathrm{A}$ has $4 p_{1} p_{2}$ orbits on $V \Gamma$, by Theorem 2.6(i), $\Gamma_{K}$ is an A/K-arc-transitive pentavalent graph of order $4 p_{1} p_{2}$, and hence $\Gamma_{K}$ satisfies the conditions in Table 3 . Since A/K is soluble, by Remark 2.8, a contradiction occurs. Hence A is insoluble. This completes the proof of the Lemma.

We now consider the case where $\mathrm{A}$ is insoluble and has no nontrivial soluble normal subgroup.

Lemma 4.2. Assume that $\mathrm{A}$ is insoluble and has no nontrivial soluble normal subgroup. Then Aut $\Gamma \cong \mathrm{J}_{1}, \operatorname{PSL}(2, p)$ or $\operatorname{PGL}(2, p)$ with $p \geq 29$. Further, if Aut $\Gamma \cong \mathrm{J}_{1}$, then $\Gamma \cong \mathcal{C}_{17556}^{i}$ satisfies the conditions in Row 1 to Row 5 of Table 1 of Theorem 1.1, where $1 \leq i \leq 5$. If Aut $\Gamma \cong \operatorname{PSL}(2, p)$ or $\operatorname{PGL}(2, p)$, then $\Gamma$ satisfies the conditions in Table 5 .

Table 5: Aut $\Gamma$ is almost simple.

\begin{tabular}{llll} 
Aut $\Gamma$ & $(\text { Aut } \Gamma)_{\alpha}$ & $\Gamma$ & Remark \\
\hline \hline $\operatorname{PSL}(2, p)$ & $\mathrm{A}_{5}$ & Example 3.4 & $p \equiv 49,79,81,111(\bmod 160)$ \\
$\operatorname{PGL}(2, p)$ & $\mathrm{A}_{5}$ & Example 3.5 & $p \equiv 9,39,41,71(\bmod 80)$ \\
$\operatorname{PSL}(2, p)$ & $\mathrm{D}_{10}$ & & $p \equiv 9,39,41,71(\bmod 80)$ \\
$\operatorname{PGL}(2, p)$ & $\mathrm{D}_{10}$ & & $p \equiv 11,19,21,29(\bmod 40)$ \\
$\operatorname{PSL}(2, p)$ & $\mathrm{D}_{20}$ & & $p \equiv 49,79,81,111(\bmod 160)$ \\
$\operatorname{PGL}(2, p)$ & $\mathrm{D}_{20}$ & & $p \equiv 9,39,41,71(\bmod 80)$
\end{tabular}

Proof. Let $N$ be the socle of A. Then $N$ is insoluble and 4 divides $|N|$. If $N$ has more than three orbits on $V \Gamma$, then by Theorem 2.6, $\Gamma_{N}$ is a pentavalent symmetric graph of odd order, a contradiction. Hence, $N$ has at most two orbits on $V \Gamma$, so $2 n$ divides $|N|$.

Assume that A has at least two minimal normal subgroups $N_{1}$ and $N_{2}$. Then by a similar argument as above, we have that $2 n$ divides both $\left|N_{1}\right|$ and $\left|N_{2}\right|$. Hence $4 n^{2}$ divides $|\mathrm{A}|=2^{11} \cdot 3^{2} \cdot 5 \cdot n$, and so $n$ divides $2^{9} \cdot 3^{2} \cdot 5$. It implies that $n=3 \cdot 5$, a contradiction with $n$ having at least three prime factors. So A has a unique minimal normal subgroup and we may write $N=S^{d}$, where $S$ is a nonabelian simple group and $d \geq 1$.

Since $p_{s}>5, p_{s}$ divides $|N|$ and $p_{s}^{2}$ does not divide $|N|$ as $|\mathrm{A}| \mid 2^{11} \cdot 3^{2} \cdot 5 \cdot p_{1} p_{2} \cdots p_{s}$, we conclude that $d=1$ and $N=S$ is a nonabelian simple group. Hence A is almost simple with socle $S$.

If $S_{\alpha}=1$, then $S$ acts regularly on $V \Gamma$. Hence $S$ is a non-abelian simple group such that $|S|=4 n$. By checking the orders of nonabelian simple groups (see [6, pp. 135-136] for example), we have that $S=\operatorname{PSL}(2, p)$ and so $\mathrm{A} \leq \operatorname{Aut}(S)=\operatorname{PGL}(2, p)$, which is impossible as $\mathrm{A}$ is transitive on $A \Gamma,|\mathrm{A}| \leq 2|S|$ and $|A \Gamma|=5|S|$. Hence $S_{\alpha} \neq 1$. Since $\Gamma$ is connected and $S \triangleleft \mathrm{A}$, we have $1 \neq S_{\alpha}^{\Gamma(\alpha)} \triangleleft \mathrm{A}_{\alpha}^{\Gamma(\alpha)}$, it follows that 5||$S_{\alpha} \mid$, we thus have $10 \cdot p_{1} p_{2} \cdots p_{s}$ divides $|S|$. 
Thus, $\operatorname{soc}(\mathrm{A})=S$ is a nonabelian simple group such that $|S| \mid 2^{11} \cdot 3^{2} \cdot 5 \cdot n$ and $10 \cdot n|| S \mid$. Hence the triple $(S,|S|, n)$ lies in Table 2 of Lemma 2.4. We will analyse all the candidates one by one in the following.

Assume $(S, n)=\left(\mathrm{J}_{1}, 3 \cdot 7 \cdot 11 \cdot 19\right)$. Then $|V \Gamma|=17556$ and $\mathrm{A} \cong \mathrm{J}_{1}$ as $\operatorname{Out}\left(\mathrm{J}_{1}\right)=1$. It then follows from Example 3.2 that $\Gamma \cong \mathcal{C}_{17556}^{i}$ satisfies the conditions in Row 1 to Row 5 of Table 1 of Theorem 1.1, where $1 \leq i \leq 5$.

Assume $(S, n)=(\mathrm{Sz}(32), 5 \cdot 31 \cdot 41)$. Since $\operatorname{Out}(\mathrm{Sz}(32)) \cong \mathbb{Z}_{5}$ (see Atlas [3] for example), $\mathrm{A} \cong \mathrm{Sz}(32)$ or $\mathrm{Sz}(32) \cdot \mathbb{Z}_{5}$, so $\left|\mathrm{A}_{\alpha}\right|=\frac{|\mathrm{A}|}{4 n}=1280$ or 6400 , which is not possible by Lemma 2.5 . Similarly, for the case $(S, n)=(\operatorname{PSL}(5,2), 3 \cdot 7 \cdot 31)$, then $\mathrm{A} \cong \operatorname{PSL}(5,2)$ or $\operatorname{PSL}(5,2) \cdot \mathbb{Z}_{2}$ as $\operatorname{Out}(\operatorname{PSL}(5,2)) \cong \mathbb{Z}_{2}$. Thus, $\left|\mathrm{A}_{\alpha}\right|=\frac{|\mathrm{A}|}{4 n}=3840$ or 7680 , which is impossible by Lemma 2.5. For the case where $(S, n)=\left(\operatorname{PSL}\left(2,2^{8}\right), 3 \cdot 17 \cdot 257\right)$, since $\mathrm{A} \cong \operatorname{PSL}\left(2,2^{8}\right) . O$, where $O \leq \operatorname{Out}\left(\operatorname{PSL}\left(2,2^{8}\right)\right) \cong \mathbb{Z}_{8}$, we have $\left|\mathrm{A}_{\alpha}\right|=\frac{|\mathrm{A}|}{4 n}=2^{k} \cdot 5$, where $6 \leq k \leq 9$, which is also impossible by Lemma 2.5. For the case where $(S, n)=$ $(\operatorname{PSU}(3,4), 3 \cdot 5 \cdot 13)$, since $\mathrm{A} \cong \operatorname{PSU}(3,4) . O$, where $O \leq \operatorname{Out}(\operatorname{PSU}(3,4)) \cong \mathbb{Z}_{4}$, we have $\left|\mathrm{A}_{\alpha}\right|=\frac{|\mathrm{A}|}{4 n}=2^{k} \cdot 5$, where $4 \leq k \leq 6$, which is impossible by Lemma 2.5 .

Assume $(S, n)=(\operatorname{PSp}(4,4), 3 \cdot 5 \cdot 17)$. Since $S \leq \mathrm{A} \leq \operatorname{Aut}(S) \cong \operatorname{PSp}(4,4) \cdot \mathbb{Z}_{4}$, we have $\left|\mathrm{A}_{\alpha}\right|=\frac{|\mathrm{A}|}{4 n}=960,1920$ or 3840 . If $\left|\mathrm{A}_{\alpha}\right|=960$ or 1920 , then by Lemma $2.5, \mathrm{~A}_{\alpha} \cong$ $\operatorname{ASL}(2,4)$ or $\mathrm{A} \Sigma \mathrm{L}(2,4)$. However, by $\operatorname{Atlas}[3], \operatorname{PSp}(4,4)$ has no subgroup isomorphic to $\operatorname{ASL}(2,4)$ and $\operatorname{PSp}(4,4) \cdot \mathbb{Z}_{2}$ has no subgroup isomorphic to $\operatorname{A\Sigma L}(2,4)$. If $\left|\mathrm{A}_{\alpha}\right|=3840$, then also by Lemma 2.5 , a contradiction occurs.

Assume $(S, n)=\left(\operatorname{PSL}\left(2,2^{6}\right), 3 \cdot 7 \cdot 13\right)$. Recall that $S$ has at most two orbits on $V \Gamma,\left|S_{\alpha}\right|=\frac{|S|}{4 n}=240$ or $\frac{|S|}{2 n}=480$. However, by Lemma 2.2, $\operatorname{PSL}\left(2,2^{6}\right)$ has no maximal subgroup with order a multiple of 240 , a contradiction occurs. Similarly, for the case $(S, n)=\left(\mathrm{J}_{2}, 3 \cdot 5 \cdot 7\right)$, then $\left|S_{\alpha}\right|=\frac{|S|}{4 n}=2880$ or $\frac{|S|}{2 n}=5760$. By Atlas [3], $\mathrm{J}_{2}$ has no maximal subgroup with order a multiple of 2880 , a contradiction also occurs.

Assume $S \cong \mathrm{M}_{23}$. Then $n=3 \cdot 7 \cdot 11 \cdot 23$ or $7 \cdot 11 \cdot 23$, and as $\operatorname{Out}\left(\mathrm{M}_{23}\right)=1$, we have $\mathrm{A}=S$ and $\left|\mathrm{A}_{\alpha}\right|=\frac{\left|\mathrm{M}_{23}\right|}{4 n}=480$ or 1440 . By Lemma 2.5 , it is impossible for the case $\left|\mathrm{A}_{\alpha}\right|=480$. For the latter case, by a direct computation using MAGMA [1], no graph $\Gamma$ exists. If $(S, n)=\left(\mathrm{M}_{22}, 7 \cdot 11 \cdot 23\right)$, as $\operatorname{Out}\left(\mathrm{M}_{22}\right) \cong \mathbb{Z}_{2}$, we have $\mathrm{A} \cong \mathrm{M}_{22}$ or $\mathrm{M}_{22} \cdot \mathbb{Z}_{2}$, so $\left|\mathrm{A}_{\alpha}\right|=\frac{|\mathrm{A}|}{4 n}=480$ or 960 , a computation by MAGMA [1] shows that no graph $\Gamma$ exists. Similarly, we can exclude the case where $(S, n)=(\operatorname{PSL}(2,25), 3 \cdot 5 \cdot 13)$ by MAGMA [1].

Assume $(S, n)=\left(\mathrm{M}_{24}, 3 \cdot 7 \cdot 11 \cdot 23\right)$ or $\left(\mathrm{J}_{1}, 3 \cdot 7 \cdot 11 \cdot 19\right)$. Since Out $\left(\mathrm{M}_{24}\right)=$ $\operatorname{Out}\left(\mathrm{J}_{1}\right)=1$, we always have $\mathrm{A}=S$. Hence $\left|\mathrm{A}_{\alpha}\right|=\frac{|\mathrm{A}|}{4 n}=11520$ or 10 . A computation by MAGMA [1] also shows that no graph $\Gamma$ exists.

Finally, assume $S \cong \operatorname{PSL}(2, p)$ with $p \geq 29$ a prime. Then $\mathrm{A} \cong \operatorname{PSL}(2, p)$ or $\operatorname{PGL}(2, p)$. By Lemma 2.2, Lemma 2.3 and Lemma 2.5, we have $\mathrm{A}_{\alpha} \cong \mathbb{Z}_{5}, \mathrm{D}_{10}, \mathrm{D}_{20}$ or $\mathrm{A}_{5}$. If $\mathrm{A}_{\alpha} \cong \mathbb{Z}_{5}$, then $\Gamma$ is an arc-regular pentavalent graph of order four times an odd square-free integer. However, by [15, Theorem 1.1], no such $\Gamma$ exists. Hence $\mathrm{A}_{\alpha} \cong \mathrm{D}_{10}, \mathrm{D}_{20}$ or $\mathrm{A}_{5}$. If $\mathrm{A}_{\alpha} \cong \mathrm{A}_{5}$, then by Lemma 2.2 and Lemma 2.3 , we have $p \equiv \pm 1$ $(\bmod 5)$. Since $\left|\mathrm{A}: \mathrm{A}_{\alpha}\right|=4 n$, we have $|\mathrm{A}|$ is divisible by 16 , but not by 32 . Since $|\mathrm{A}|=|\operatorname{PSL}(2, p)|=\frac{p(p-1)(p+1)}{2}$ or $|\operatorname{PGL}(2, p)|=p(p-1)(p+1)$, we have $p \equiv \pm 15$ $(\bmod 32)$ for $\mathrm{A} \cong \operatorname{PSL}(2, p)$ or $p \equiv \pm 7(\bmod 16)$ for $\mathrm{A} \cong \operatorname{PGL}(2, p)$. Since $p \equiv \pm 1$ $(\bmod 5)$, we have $p \equiv 49,79,81,111(\bmod 160)$ for $\mathrm{A} \cong \operatorname{PSL}(2, p)$ or $p \equiv 9,39,41,71$ $(\bmod 80)$ for $\mathrm{A} \cong \mathrm{PGL}(2, p)$. These graphs are constructed in Example 3.4 and Example 3.5. Similarly, if $\mathrm{A}_{\alpha} \cong \mathrm{D}_{10}$ or $\mathrm{D}_{20}$, then $p$ satisfies the condition in Table 5. This completes the proof of the Lemma. 
We next assume that A has a nontrivial soluble normal subgroup. Let $N$ be a soluble minimal normal subgroup of A. Then there exists a prime $r \mid 4 n$ such that $N \cong \mathbb{Z}_{r}^{d}$. Further, $N$ has at least three orbits on $V \Gamma$. It follows from Theorem 2.6 that $N$ is semi-regular on $V \Gamma$, and so $|N|=\left|\mathbb{Z}_{r}\right|^{d}|| V \Gamma \mid=4 n$. If $d \geq 2$, then $(r, d)=(2,2)$. It follows that $\Gamma_{N}$ is an arc-transitive graph of odd order, a contradiction. Hence $d=1, N=\mathbb{Z}_{r}$. The next lemma consider the case where $r=2$.

Lemma 4.3. Assume that $\mathrm{A}$ is insoluble and has a soluble minimal normal subgroup $N=$ $\mathbb{Z}_{2}$. Then one of the following statements holds:

(1) Aut $\Gamma \cong \operatorname{PSL}(2, p) \times \mathbb{Z}_{2}$ or $\operatorname{PGL}(2, p) \times \mathbb{Z}_{2}$, where $p \geq 29$ is a prime. Furthermore, $\Gamma$ satisfies the conditions in Table 6.

(2) Aut $\Gamma \cong \operatorname{PSL}(2,25) \times \mathbb{Z}_{2}$ and $\Gamma$ is isomorphic to $\mathcal{C}_{780}^{i}$ in Table 1 , where $1 \leq i \leq 3$.

(3) Aut $\Gamma \cong \mathrm{J}_{1} \times \mathbb{Z}_{2}$ and $\Gamma$ is isomorphic to $\mathcal{C}_{5852}$ in Table 1 .

Table 6: Aut $\Gamma$ has a normal subgroup isomorphic to $\mathbb{Z}_{2}$.

\begin{tabular}{llll} 
Aut $\Gamma$ & $(\text { Aut } \Gamma)_{\alpha}$ & $\Gamma$ & Remark \\
\hline \hline $\operatorname{PSL}(2, p) \times \mathbb{Z}_{2}$ & $\mathrm{~A}_{5}$ & Example 3.6 & $p \equiv 9,39,41,71(\bmod 80)$ \\
$\operatorname{PGL}(2, p) \times \mathbb{Z}_{2}$ & $\mathrm{~A}_{5}$ & Example 3.7 & $p \equiv 11,19,21,29(\bmod 40)$ \\
$\operatorname{PSL}(2, p) \times \mathbb{Z}_{2}$ & $\mathrm{D}_{10}$ & & $p \equiv 11,19,21,29(\bmod 40)$ \\
$\operatorname{PSL}(2, p) \times \mathbb{Z}_{2}$ & $\mathrm{D}_{20}$ & & $p \equiv 9,39,41,71(\bmod 80)$ \\
$\operatorname{PGL}(2, p) \times \mathbb{Z}_{2}$ & $\mathrm{D}_{20}$ & & $p \equiv 11,19,21,29(\bmod 40)$
\end{tabular}

Proof. Since $N$ has more than three orbits on $V \Gamma$, then by Theorem $2.6, \Gamma_{N}$ is an $\mathrm{A} / N$ arc-transitive pentavalent graph of order $\bar{n}=2 n$. It follows that $\Gamma_{N}$ is isomorphic to one of the graphs in Lemma 2.9. Since $\mathrm{A} / N \leq \mathrm{Aut} \Gamma_{N}$ and $\mathrm{A} / N$ is insoluble, we have that Aut $\Gamma_{N}$ is insoluble and so Aut $\Gamma_{N} \cong \operatorname{PSL}(2, p), \operatorname{PGL}(2, p), \operatorname{PSL}(2,25)$ or $\mathrm{J}_{1}$. Let $\overline{\mathrm{A}}:=$ Aut $\bar{\Gamma}$.

Suppose that $\overline{\mathrm{A}} \cong \operatorname{PSL}(2, p)$ or $\operatorname{PGL}(2, p)$. Since $\mathrm{A} / N$ is insoluble, by Lemma 2.2 and Lemma 2.3, $\mathrm{A} / N$ is isomorphic to $\mathrm{A}_{5}, \operatorname{PSL}(2, p)$ or $\operatorname{PGL}(2, p)$. If $\mathrm{A} / N \cong \mathrm{A}_{5}$, then since $\Gamma_{N}$ is an A/N-arc-transitive pentavalent graph of order $\bar{n}=2 n$, we have $2 n \cdot 5|| \mathrm{A}_{5} \mid$. It implies that $n$ divides 6 , a contradiction with $n$ having at least three odd prime factors. Thus, $\mathrm{A} / N$ is isomorphic to $\operatorname{PSL}(2, p)$ or $\operatorname{PGL}(2, p)$. Therefore, $\mathrm{A} \cong N$. $\operatorname{PSL}(2, p)$ or $N . \operatorname{PGL}(2, p)$, that is, $\mathrm{A} \cong \operatorname{PSL}(2, p) \times \mathbb{Z}_{2}, \operatorname{SL}(2, p), \operatorname{PGL}(2, p) \times \mathbb{Z}_{2}$ or $\operatorname{SL}(2, p) \cdot \mathbb{Z}_{2}$. Assume first that $\mathrm{A} \cong \mathrm{SL}(2, p)$. Note that $\mathrm{SL}(2, p)$ has a unique central involution. Then by Lemma $2.5, \mathrm{~A}_{\alpha} \cong \mathbb{Z}_{5}$. It follows that $|V \Gamma|=\left|\mathrm{A}: \mathrm{A}_{\alpha}\right|$ is divisible by 8 as $|\operatorname{SL}(2, p)|$ is divisible by 8 , a contradiction. Assume next that $\mathrm{A} \cong \mathrm{SL}(2, p) \cdot \mathbb{Z}_{2}$. Then A contains a normal subgroup $H$ isomorphic to $\operatorname{SL}(2, p)$. Since 8||$H \mid$, we have $H_{\alpha} \neq 1$. By Theorem 2.6, $H$ has at most two orbits on $V \Gamma$ and so $\frac{\left|\mathrm{A}_{\alpha}\right|}{\left|H_{\alpha}\right|} \mid 2$. If $H$ is transitive on $V \Gamma$, then $H$ is arc-transitive. A similar argument with the case $\mathrm{A} \cong \mathrm{SL}(2, p)$, a contradiction occurs. Therefore, $H$ has two orbits on $V \Gamma$ and so $H_{\alpha}=\mathrm{A}_{\alpha}$. Since $H$ has a unique central involution, by Lemma $2.5, \mathrm{~A}_{\alpha} \cong \mathbb{Z}_{5}$, it follows that $|V \Gamma|=\left|\mathrm{A}: \mathrm{A}_{\alpha}\right|$ is divisible by 16 , a contradiction. Therefore, $\mathrm{A} \cong \operatorname{PSL}(2, p) \times \mathbb{Z}_{2}$ or $\operatorname{PGL}(2, p) \times \mathbb{Z}_{2}$ in this case. By a similar 
argument as for the case $\mathrm{A} \cong \operatorname{PSL}(2, p)$ (the last paragraph in the proof of Lemma 4.2), we have that $\Gamma$ satisfies the condition in Table 6 . Note that since 16 divides $\left|\operatorname{PGL}(2, p) \times \mathbb{Z}_{2}\right|$ and $\left|\mathrm{A}: \mathrm{A}_{\alpha}\right|=4 n$, we have $\left(\mathrm{A}, \mathrm{A}_{\alpha}\right) \neq\left(\operatorname{PGL}(2, p) \times \mathbb{Z}_{2}, \mathrm{D}_{10}\right)$.

Suppose that $\overline{\mathrm{A}} \cong \operatorname{PSL}(2,25)$. Since $\Gamma_{N}$ is $\mathrm{A} / N$-arc-transitive, we have that $5 \cdot 390|| \mathrm{A} / N \mid$. By checking the maximal subgroup of $\operatorname{PSL}(2,25)$ (see Atlas [3] for example), we have that $\mathrm{A} / N=\overline{\mathrm{A}} \cong \operatorname{PSL}(2,25)$. It follows that $\mathrm{A} \cong \mathrm{SL}(2,25)$ or $\operatorname{PSL}(2,25) \times \mathbb{Z}_{2}$. If $\mathrm{A} \cong \operatorname{PSL}(2,25) \times \mathbb{Z}_{2}$, then by Example $3.2, \Gamma \cong \mathcal{C}_{780}^{i}$ in Table 1 , where $1 \leq i \leq 3$. If $\mathrm{A} \cong \mathrm{SL}(2,25)$, then by MAGMA [1], no graph $\Gamma$ exists.

Suppose that $\overline{\mathrm{A}} \cong \mathrm{J}_{1}$. Similarly, since $\Gamma_{N}$ is $\mathrm{A} / N$-arc-transitive, we have that $5 \cdot 2926|| \mathrm{A} / N \mid$. By checking the maximal subgroup of $\mathrm{J}_{1}$ (see Atlas [3] for example), we have that $\mathrm{A} / N=\overline{\mathrm{A}} \cong \mathrm{J}_{1}$. Since the Schur multiplier of $\mathrm{J}_{1}$ is $\mathbb{Z}_{1}, \mathrm{~A} \cong N . \mathrm{J}_{1} \cong \mathrm{J}_{1} \times \mathbb{Z}_{2}$. By Example 3.2, $\Gamma \cong \mathcal{C}_{5852}$ in Table 1 .

Finally, suppose that $r>2$. We first prove the following lemma.

Lemma 4.4. Let $\Sigma$ be a graph. Assume that $\Sigma$ is isomorphic to one of the graphs appearing in Lemma 2.7, in Lemma 4.2 or in Lemma 4.3. If $M$ is an arc-transitive subgroup of Aut $\Sigma$, then $M$ contains the derived subgroup of Aut $\Sigma$.

Proof. Let $\Sigma$ be a graph and isomorphic to one of the graphs appearing in Lemma 2.7, in Lemma 4.2 or in Lemma 4.3. Let $M$ be an arc-transitive subgroup of $B=$ Aut $\Sigma$. Then $B=M B_{\alpha \beta}$, where $(\alpha, \beta) \in A \Sigma$. In particular, $m:=|B: M|$ divides $\left|B_{\alpha \beta}\right|$. Assume first that $\Sigma$ is isomorphic to one of the graphs appearing in Lemma 2.7. Then, in the first three rows of Table 3, we have that $M$ has index at most two, and for the fourth row $M$ has index at most four, so in particular, $M$ contains $B^{\prime}$. For the last two rows, we have that $m \mid 12$. Since there is no faithful representation of $B$ in degree $m$ for $2<m \leq 12$, we have $1 \leq m \leq 2$ and so $M$ also contains $B^{\prime}$.

Now assume that $\Sigma$ is isomorphic to one of the graphs appearing in Lemma 4.2 or in Lemma 4.3. Then $B$ is isomorphic to one of the groups $\operatorname{PSL}(2, p), \operatorname{PGL}(2, p), \operatorname{PSL}(2, p) \times$ $\mathbb{Z}_{2}, \operatorname{PGL}(2, p) \times \mathbb{Z}_{2}, \mathrm{~J}_{1}, \mathrm{~J}_{1} \times \mathbb{Z}_{2}$ or $\operatorname{PSL}(2,25) \times \mathbb{Z}_{2}$ with $p \geq 29$. If $B \cong \mathrm{J}_{1}$, then $M$ has index at most two. If $B \cong \mathrm{J}_{1} \times \mathbb{Z}_{2}$, then $M$ has index at most 12 . If $B \cong \operatorname{PSL}(2,25) \times \mathbb{Z}_{2}$, then $M$ has index at most four. For these three cases, by a similar argument as above, we also have $M$ contains $B^{\prime}$. If $B \cong \operatorname{PSL}(2, p)$, then since $p \mid n$ and $20 n|| M \mid$, by Lemma 2.2, $M \leq \mathbb{Z}_{p}: \mathbb{Z}_{\frac{p-1}{2}}$ or $M=B \cong \operatorname{PSL}(2, p)$. If $M \leq \mathbb{Z}_{p}: \mathbb{Z}_{\frac{p-1}{2}}$, then $M \cong \mathbb{Z}_{p}: \mathbb{Z}_{l}$ for some $l \mid \frac{p-1}{2}$. Thus, $M$ has a normal subgroup, say $S \cong \mathbb{Z}_{p}$, which has more than three orbits on $V \Sigma$. It then follows from Theorem 2.6 that the normal quotient graph $\Sigma_{S}$ is $M / S$ arc-transitive, a contradiction occurs as $M / S \cong \mathbb{Z}_{l}$ is cyclic. Hence, $M \not \mathbb{Z}_{p}: \mathbb{Z}_{\frac{p-1}{2}}$ and so $M=B^{\prime} \cong \operatorname{PSL}(2, p)$. If $B \cong \operatorname{PGL}(2, p)$, then since $20 n|| M \mid$, by Lemma 2.3, $\mathrm{M} \leq \mathbb{Z}_{p}: \mathbb{Z}_{p-1}, M \leq \operatorname{PSL}(2, p)$ or $M=B \cong \operatorname{PGL}(2, p)$. With a similar argument, we can conclude that $M \geq B^{\prime} \cong \operatorname{PSL}(2, p)$. Similarly, we can further show that $M \geq B^{\prime} \cong$ $\operatorname{PSL}(2, p)$ for the case $B \cong \operatorname{PSL}(2, p) \times \mathbb{Z}_{2}$ or $\operatorname{PGL}(2, p) \times \mathbb{Z}_{2}$.

Now assume that A has a soluble minimal normal subgroup $N=\mathbb{Z}_{r}$ for $r>2$.

Lemma 4.5. Assume that A has a soluble minimal normal subgroup $N=\mathbb{Z}_{r}$ for $r>2$. Then the normal quotient $\Gamma_{N}$ is not isomorphic to any graph appearing in Lemma 2.7, Lemma 4.2 or Lemma 4.3. 
Proof. Suppose to the contrary that $\Gamma_{N}$ is isomorphic to one of the graphs appearing in Lemma 2.7, Lemma 4.2 or Lemma 4.3. Let $M / N=\left(\text { Aut } \Gamma_{N}\right)^{\prime}$, and let $\Omega:=\{\operatorname{PSL}(2, p)$, $\left.\mathrm{J}_{1}, \operatorname{PSL}(2,25), \mathrm{A}_{5}\right\}$. By checking the graphs appearing in Lemma 2.7, in Lemma 4.2 or in Lemma 4.3, we have that Aut $\Gamma_{N}$ is isomorphic to one of the groups $\operatorname{PSL}(2, p), \operatorname{PGL}(2, p)$, $\operatorname{PSL}(2, p) \times \mathbb{Z}_{2}, \operatorname{PGL}(2, p) \times \mathbb{Z}_{2}, \mathrm{~J}_{1}, \mathrm{~J}_{1} \times \mathbb{Z}_{2}, \operatorname{PSL}(2,25) \times \mathbb{Z}_{2}$ or $\mathrm{A}_{5} \times \mathrm{D}_{10}$. Thus, $M / N$ is isomorphic to one of the groups in $\Omega$. Since the order of the Schur multiplier of a group in $\Omega$ is less than or equal to 2 (see [10, Theorem 7.1.1] for $\operatorname{PSL}(2, p)$ and Atlas [3] for the others) and $r>2$, we have that $M^{\prime} \in \Omega$.

By Theorem 2.6, A/N $\leq$ Aut $\Gamma_{N}$ is transitive on $A \Gamma_{N}$. It follows from Lemma 4.4 that $\mathrm{A} / N$ contains the derived subgroup of Aut $\Gamma_{N}$, that is, $M / N \leq \mathrm{A} / N$. Since $M / N \unlhd$ Aut $\Gamma_{N}$, we have $M / N \unlhd \mathrm{A} / N$. Therefore, $M^{\prime}$ char $M \unlhd \mathrm{A}$, it implies that $M^{\prime} \unlhd$ A. If $M^{\prime}$ has more than three orbits on $V \Gamma$, then by Theorem 2.6, $\Gamma_{M^{\prime}}$ is a pentavalent symmetric graph of odd order, a contradiction. Thus, $M^{\prime}$ has at most two orbits on $V \Gamma$ and so $2 n$ divides $\left|M^{\prime}\right|$. Let $\overline{\mathrm{A}}:=$ Aut $\Gamma_{N}, \bar{n}:=\frac{n}{r}$ and $\bar{M}:=M / N$. Then $M^{\prime} \cong \bar{M}$.

Let $\rho$ be the bijection from the orbits of $M^{\prime}$ on $V \Gamma$ to the orbits of $\bar{M}$ on $V \Gamma_{N}$ defined by:

$$
\alpha^{M^{\prime}} \rightarrow \delta^{\bar{M}}, \quad \text { where } \quad \alpha \in V \Gamma \text { and } \delta=\alpha^{N} \in V \Gamma_{N} .
$$

Then we can conclude that, for some $k \in\{2,4\},\left|M^{\prime}:\left(M^{\prime}\right)_{\alpha}\right|=k n$ and $\left|\bar{M}: \bar{M}_{\delta}\right|=\frac{k n}{r}$. It gives $\left|\left(M^{\prime}\right)_{\alpha}\right| r=\left|\bar{M}_{\delta}\right|$. Since $\left|\bar{M}_{\delta}\right||| \overline{\mathrm{A}}_{\delta} \mid$ and $\left|\overline{\mathrm{A}}_{\delta}\right| \mid 2^{9} \cdot 3^{2} \cdot 5$, we have $\left|\bar{M}_{\delta}\right| \mid 2^{9} \cdot 3^{2} \cdot 5$ and so $r=3$ or 5 .

Assume first that $r=5$. Since $\Gamma$ is connected and $1 \neq M_{\alpha}^{\prime} \triangleleft \mathrm{A}_{\alpha}$, we have $1 \neq$ $M_{\alpha}^{\prime \Gamma(\alpha)} \triangleleft \mathrm{A}_{\alpha}^{\Gamma(\alpha)}$, it follows that 5||$M_{\alpha}^{\prime} \mid$. Therefore, $5^{2}|| \bar{M}_{\delta} \mid$, a contradiction.

Now assume that $r=3$. Since $\bar{M} \cong M^{\prime}$ has at most two orbits on $V \Gamma_{N}$ (if $\operatorname{not}\left(\Gamma_{N}\right)_{\bar{M}}$ is a pentavalent symmetric graph of odd order, a contradiction), we have that $\left|\bar{M}: \bar{M}_{\delta}\right|=$ $2 \bar{n}$ or $4 \bar{n}$, where $\delta \in V \bar{\Gamma}$. Now $2 n$ divides $|\bar{M}|$ and $\left|\bar{M}: \bar{M}_{\delta}\right|=\frac{2 n}{r}$ or $\frac{4 n}{r}$. It implies that $r=3$ divides $\bar{M}_{\delta}$. Therefore 3||$\overline{\mathrm{A}}_{\delta} \mid$. By Lemma 2.5, $\overline{\mathrm{A}}_{\delta}$ is insoluble, because $\left|\overline{\mathrm{A}}_{\delta}\right|$ does not divide 80 , forcing that $\bar{M}_{\delta}$ is insoluble. Recall that $\bar{M} \cong M^{\prime} \in \Omega$. If $\bar{M} \cong \operatorname{PSL}(2, p)$, then by Lemma $2.2, \bar{M}_{\delta} \cong \mathrm{A}_{5}$. Hence $M_{\alpha}^{\prime} \leq\left(M^{\prime} N\right)_{\alpha} \cong\left(M^{\prime} N / N\right)_{\delta}=\bar{M}_{\delta} \cong \mathrm{A}_{5}$ by Theorem 2.6(ii). Note that $\left|M_{\alpha}^{\prime}\right|=20$, it contradicts that $\mathrm{A}_{5}$ has no subgroup of order 20 . If $\bar{M} \cong \mathrm{J}_{1}$, then $\Gamma_{N} \cong \mathcal{C}_{5852}$ or $\mathcal{C}_{17556}^{i}$ in Table 1 , where $1 \leq i \leq 5$. If $\Gamma_{N} \cong \mathcal{C}_{17556}^{i}$, then $\overline{\mathrm{A}}_{\delta} \cong \mathrm{D}_{10}$ is soluble, a contradiction. If $\Gamma_{N} \cong \mathcal{C}_{5852}$, then $\bar{M}_{\delta}=\overline{\mathrm{A}}_{\delta} \cong \mathrm{A}_{5}$. A similar argument with the case $\bar{M} \cong \mathrm{PSL}(2, p)$ leads to a contradiction. If $\bar{M} \cong \mathrm{A}_{5}$, then $\Gamma_{N} \cong \mathcal{C}_{60}$ in Table 3 and $\overline{\mathrm{A}}_{\delta} \cong \mathrm{D}_{10}$ is soluble, a contradiction. If $\bar{M} \cong \operatorname{PSL}(2,25)$, then $\Gamma_{N} \cong \mathcal{C}_{780}^{1}, \mathcal{C}_{780}^{2}$ or $\mathcal{C}_{780}^{3}$ in Table 1 and $\overline{\mathrm{A}}_{\delta} \cong \mathrm{F}_{20}$ is soluble, also a contradiction.

The final lemma completes the proof of Theorem 1.1.

Lemma 4.6. Assume A is insoluble. Then A has no soluble minimal normal subgroup isomorphic to $\mathbb{Z}_{r}$ with $r>2$.

Proof. Suppose that, on the contrary, A has a soluble minimal normal subgroup $N=\mathbb{Z}_{r}$ with $r>2$. We prove the lemma by induction on the order of $\Gamma$.

Assume first that $n=p q t$ has three prime factors. (Note that, by Table 3, the conclusion of Lemma 4.6 does not hold for $n=p q$.) Without loss of generality, we may assume that $r=t$. Then $\Gamma_{N}$ is a pentavalent symmetric graph of order $4 p q$. By Lemma $2.7, \Gamma_{N}$ is isomorphic to one of the graphs in Table 3, which contradicts to Lemma 4.5.

Assume next that $n$ has at least four prime factors. Note that Aut $\Gamma_{N}$ is insoluble. If Aut $\Gamma_{N}$ has no nontrivial soluble normal subgroup, then $\Gamma_{N}$ is isomorphic to one of the 
graphs in Lemma 4.2, which contradicts to Lemma 4.5. If Aut $\Gamma_{N}$ has a soluble minimal normal subgroup $\bar{N}$, then we can also conclude that $\bar{N} \cong \mathbb{Z}_{f}$ with $f$ a prime. If $f>2$, then by induction, no such $\Gamma_{N}$ exists, a contradiction. If $f=2$, then $\Gamma_{N}$ is isomorphic to one of the graphs appearing in Lemma 4.3, which also contradicts to Lemma 4.5. This completes the proof of the Lemma.

\section{References}

[1] W. Bosma, J. Cannon and C. Playoust, The Magma algebra system I: The user language, J. Symbolic Comput. 24 (1997), 235-265, doi:10.1006/jsco.1996.0125.

[2] P. J. Cameron, G. R. Omidi and B. Tayfeh-Rezaie, 3-designs from $\operatorname{PGL}(2, q)$, Electron. J. Combin. 13 (2006), \#R50, http: / /www. combinatorics.org/ojs/index.php/ eljc/article/view/v13ilr50.

[3] J. H. Conway, R. T. Curtis, S. P. Norton, R. A. Parker and R. A. Wilson, Atlas of Finite Groups, Oxford University Press, Eynsham, 1985.

[4] L. E. Dickson, Linear Groups: With an Exposition of the Galois Field Theory, Dover Publications, New York, 1958.

[5] Y.-Q. Feng, J.-X. Zhou and Y.-T. Li, Pentavalent symmetric graphs of order twice a prime power, Discrete Math. 339 (2016), 2640-2651, doi:10.1016/j.disc.2016.05.008.

[6] D. Gorenstein, Finite Simple Groups, University Series in Mathematics, Plenum Publishing, New York, 1982.

[7] S.-T. Guo and Y.-Q. Feng, A note on pentavalent s-transitive graphs, Discrete Math. 312 (2012), 2214-2216, doi:10.1016/j.disc.2012.04.015.

[8] S.-T. Guo, J.-X. Zhou and Y.-Q. Feng, Pentavalent symmetric graphs of order $12 p$, Electron. J. Combin. 18 (2011), \#P233, http: / /www. combinatorics.org/ojs/index.php/ eljc/article/view/v18ilp233.

[9] X.-H. Hua, Y.-Q. Feng and J. Lee, Pentavalent symmetric graphs of order 2pq, Discrete Math. 311 (2011), 2259-2267, doi:10.1016/j.disc.2011.07.007.

[10] G. Karpilovsky, The Schur Multiplier, volume 2 of London Mathematical Society Monographs New Series, The Clarendon Press, Oxford, 1987.

[11] C. H. Li, Z. P. Lu and G. Wang, Arc-transitive graphs of square-free order and small valency, Discrete Math. 339 (2016), 2907-2918, doi:10.1016/j.disc.2016.06.002.

[12] C. H. Li and J. Pan, Finite 2-arc-transitive abelian Cayley graphs, European J. Combin. 29 (2008), 148-158, doi:10.1016/j.ejc.2006.12.001.

[13] Y. Li and Y.-Q. Feng, Pentavalent one-regular graphs of square-free order, Algebra Colloq. 17 (2010), 515-524, doi:10.1142/s1005386710000490.

[14] J. Pan, B. Ling and S. Ding, On prime-valent symmetric graphs of square-free order, Ars Math. Contemp. 15 (2018), 53-65, doi:10.26493/1855-3974.1161.3b9.

[15] J. Pan and Y. Liu, There exist no arc-regular prime-valent graphs of order four times an odd square-free integer, Discrete Math. 313 (2013), 2575-2581, doi:10.1016/j.disc.2013.08.009.

[16] J. Pan, B. Lou and C. Liu, Arc-transitive pentavalent graphs of order 4pq, Electron. J. Combin. 20 (2013), \#P36, http: / / www. combinatorics.org/ojs/index.php/eljc/ article/view/v20ilp36.

[17] C. E. Praeger, An O'Nan-Scott theorem for finite quasiprimitive permutation groups and an application to 2-arc transitive graphs, J. London Math. Soc. 47 (1993), 227-239, doi:10.1112/ jlms/s2-47.2.227. 
[18] M. Suzuki, Group Theory II, volume 248 of Grundlehren der Mathematischen Wissenschaften, Springer-Verlag, New York, 1986, translated from the Japanese.

[19] D.-W. Yang, Y.-Q. Feng and J.-L. Du, Pentavalent symmetric graphs of order 2pqr, Discrete Math. 339 (2016), 522-532, doi:10.1016/j.disc.2015.09.009.

[20] J.-X. Zhou and Y.-Q. Feng, On symmetric graphs of valency five, Discrete Math. 310 (2010), 1725-1732, doi:10.1016/j.disc.2009.11.019. 